\title{
Linfoma de Hodgkin cutáneo maligno: reporte de caso y revisión de la literatura
}

\author{
Vanessa Pérez-Carrillo; Delsy Yurledy del Rio-Cobaleda²; Ana Cristina Ruiz-Suárez; \\ Andrea Laverde-Walter ${ }^{4}$ \\ RESUMEN
}

El linfoma de Hodgkin (LH) es una neoplasia linfoide en la que se mezclan células malignas de Hodgkin y de Reed-Sternberg con una población heterogénea de células inflamatorias no neoplásicas como linfocitos, células plasmáticas, eosinófilos e histiocitos. El LH se presenta de forma típica como una linfadenopatía indolora. La afectación cutánea puede ser inespecífica o específica. En las formas inespecíficas, las manifestaciones clínicas son variadas y no hay evidencia histológica del LH en la piel. La forma específica es inusual, entre el o,5\% y el $7,5 \%$ de los casos, en la cual la piel es infiltrada por células neoplásicas; se observa en pacientes en estado avanzado de la enfermedad y representa mal pronóstico. El compromiso cutáneo específico del LH puede ser primario o secundario a la enfermedad ganglionar. Se describe el caso de un hombre de 83 años con LH clásico y compromiso cutáneo específico, demostrado por histopatología y confirmado por inmunofenotipificación.

PALABRAS CLAVE: linfoma de Hodgkin cutáneo; neoplasia; célula de Reed-Sternberg; inmunohistoquímica.

\section{MALIGNANT CUTANEOUS HODGKIN LYMPHOMA: CASE REPORT AND LITERATURE REVIEW}

\section{SUMMARY}

Hodgkin lymphoma (HL) is a lymphoid neoplasm in which malignant Hodgkin and Reed-Sternberg cells are mixed with a heterogeneous population of non-neoplastic inflammatory cells such as lymphocytes, plasma cells, eosinophils and histiocytes. LH typically presents as painless lymphadenopathy. The skin involvement can be nonspecific or specific; in the nonspecific forms the clinical manifestations are varied and there is no histological evidence of HL in the skin. The specific form is unusual, between $0.5 \%$ to $7.5 \%$ of cases, in which the skin is infiltrated by neoplastic cells, it is observed in patients in an advanced stage of the disease and represents a poor prognosis. Specific HL skin involvement may be primary or secondary to lymph node disease. We describe the case of an 83-year-old man with classic HL and specific cutaneous involvement, demonstrated by histopathology and confirmed by immunophenotyping.

KEY WORDS: Cutaneous Hodgkin lymphoma; Neoplasm; Reed-Sternberg cell; Immunohistochemistry.

\footnotetext{
1. Médica general, Universidad Pontificia Bolivariana, Medellín, Colombia. ORCID https://orcid.org/oooo-0002-960o-8597

2. Médica dermatóloga, Hospital Pablo Tobón Uribe, Medellín, Colombia. ORCID https://orcid.org/oooo-0002-8137-2695

3. Médica dermatopatóloga, Hospital Pablo Tobón Uribe, Medellín, Colombia. ORCID https://orcid.org/oooo-0002-0603-4063

4. Médica dermatóloga, Universidad CES, Medellín, Colombia. ORCID https://orcid.org/oooo-0002-0004-5933
}

Correspondencia: Vanesa Pérez-Carrillo; email: vanepc_19@hotmail.com

Recibido: 06/10/19; aceptado: 16/05/20

Cómo citar: Pérez-Carrillo V, del Rio-Cobaleda DY, Ruiz-Suárez AC, Laverde-Walter A. Linfoma de hodgkin Rev Asoc Colomb Dermatol. 2020;28(2): 184-189. DOI:

Financiación: ninguna, conflictos de interés: ninguno 


\section{CASO CLÍNICO}

Se presenta el caso de un hombre de 83 años con diagnóstico reciente de linfoma de Hodgkin clásico, subtipo esclerosis nodular, en estadio IVB, con compromiso cervical, intraparotídeo, supraclavicular, retropectoral y axilar, con antecedentes de diabetes mellitus tipo 2, hipertensión arterial e hipotiroidismo.

Fue evaluado por dermatología por lesiones cutáneas generalizadas muy pruriginosas de 1 o 2 meses de evolución, que iniciaron en el cuello con posterior extensión a las extremidades y el tronco.
Al examen físico se evidenció un nódulo eritematovioláceo, firme, de bordes irregulares con descamación moderada y edema periférico, de 1,5 cm de diámetro, en región retroauricular izquierda (figura 1A); lesiones similares más pequeñas en ramas mandibulares y surco nasogeniano derecho; además, placas eritematosas, infiltradas, descamativas, con bordes irregulares con hipertermia, localizadas en las extremidades superiores, espalda, tórax anterior y abdomen (figura 1B). Presentaba xerosis generalizada y excoriaciones por rascado. También presentaba adenopatías induradas, adheridas, mayores de $2 \mathrm{~cm}$, en la región supraclavicular y el cuello.

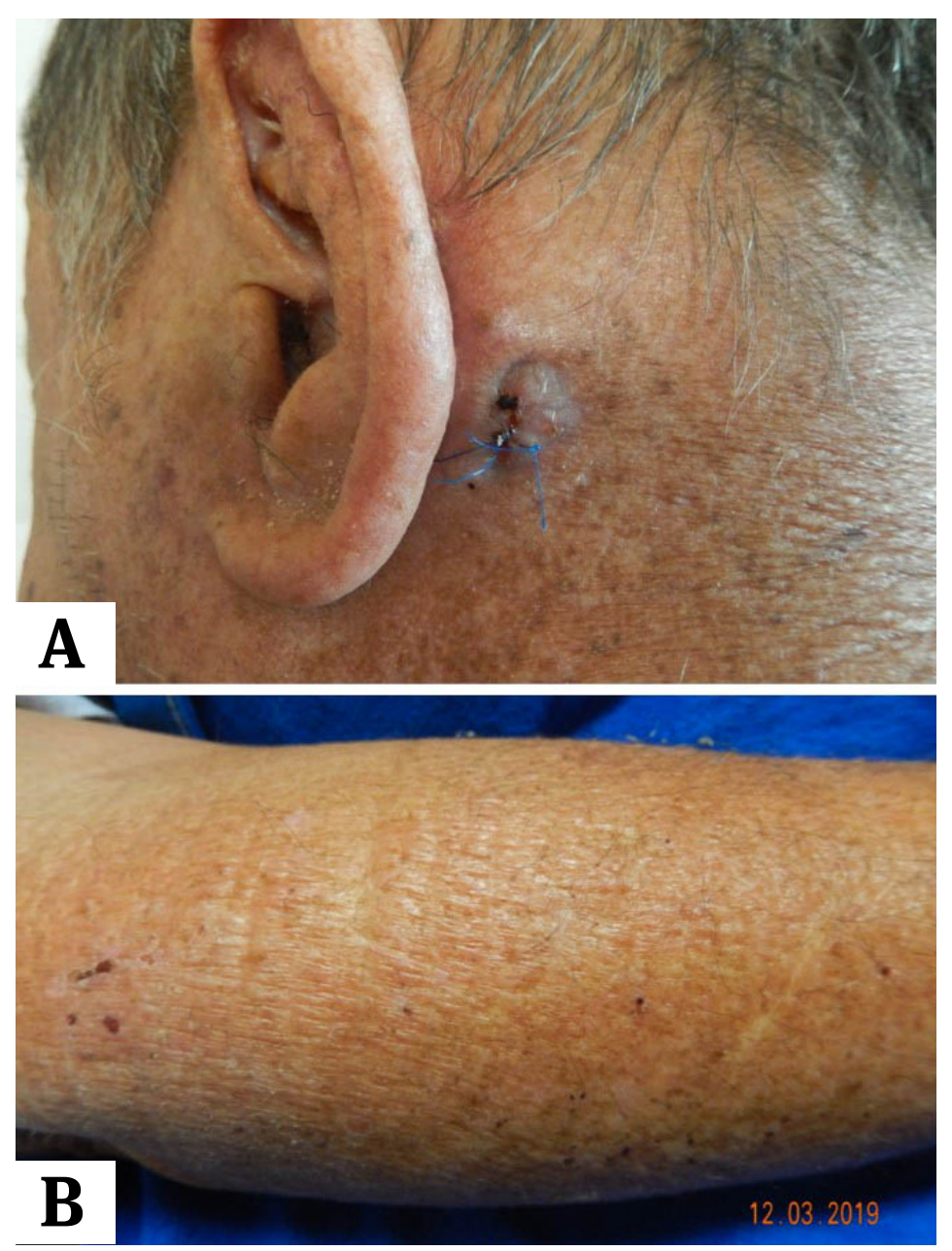

Figura 1. A) Región retroauricular izquierda: nódulo eritematoso, firme, de bordes irregulares y edema. B) Antebrazo izquierdo: xerosis y placas infiltradas. Excoriaciones por rascado. 
En la biopsia de piel que se obtuvo de las lesiones en el antebrazo y la región retroauricular izquierda se evidenció una epidermis sin cambios y en la dermis, infiltrado nodular compuesto por linfocitos pequeños, algunos eosinófilos y células grandes mononucleares o binucleadas con nucléolo prominente y citoplasma amplio, muy sugestivas de células de Hodgkin y ReedSternberg (figura 2). En la inmunofenotipificación por inmunohistoquímica se encontró fuerte expresión para CD30 y CD15 y más débil para PAX 5 y fueron negativas para CD20, CD3, CD45, ALK-1 y EMA. Estos hallazgos confirmaron el diagnóstico de compromiso cutáneo específico y secundario del linfoma de Hodgkin (figura 3). En la biopsia de ganglio linfático se observaba presencia de linfocitos atípicos grandes tipo Reed-Stemberg/Hodgkin que expresan CD3o, CD15, PAX débil y Fascina; además eran negativas para CD 20, LMP1, BOB1 y CD 45. Abundantes linfocitos $\mathrm{T}$ de tamaño pequeño que expresan $\mathrm{CD}_{\text {y }} \mathrm{CD}_{5}$, con lo que se hizo el diagnóstico inicial de linfoma de Hodgkin clásico.

El paciente recibió tratamiento con quimioterapia ABVD (adriamicina, vinblastina, bleomicina y decarbacina) con mejoría de las lesiones cutáneas y sin complicaciones. En el momento, continúa seguimiento clínico por servicio de hematología.

\section{DISCUSIÓN}

En Estados Unidos y otras regiones económicamente desarrolladas, el LH representa aproximadamente el $10 \%$ de todos los linfomas, el o,6\% de todos los cánceres y el o,2\% de todas las muertes por cáncer ${ }^{(1)}$. El LH, descrito inicialmente por el patólogo británico Thomas Hodgkin en 1832, es una neoplasia linfoide compuesta predominantemente por células B, en las que se mezclan células malignas de Hodgkin (células mononucleadas atípicas) y células de Reed-Sternberg (multinucleadas) con una población heterogénea de células inflamatorias no neoplásicas como linfocitos, células plasmáticas, eosinófilos e histiocitos ${ }^{(2,3)}$. La infección por el virus de Epstein-Barr (VEB) se ha relacionado como un factor de riesgo para la enfermedad y su detección varía con la zona geográfica, el estado de inmunocompetencia del paciente y con el subtipo histológico ${ }^{(4)}$.

Existen dos tipos principales de LH: el LH clásico y el LH no clásico; este último denominado de predominio linfocítico nodular. El LH clásico es la variedad más común y corresponde aproximadamente al 90\% de LH; según la clasificación europea-americana revisada del linfoma (REAL, por sus siglas en inglés) se divide en cuatro subtipos histológicos basándose en la
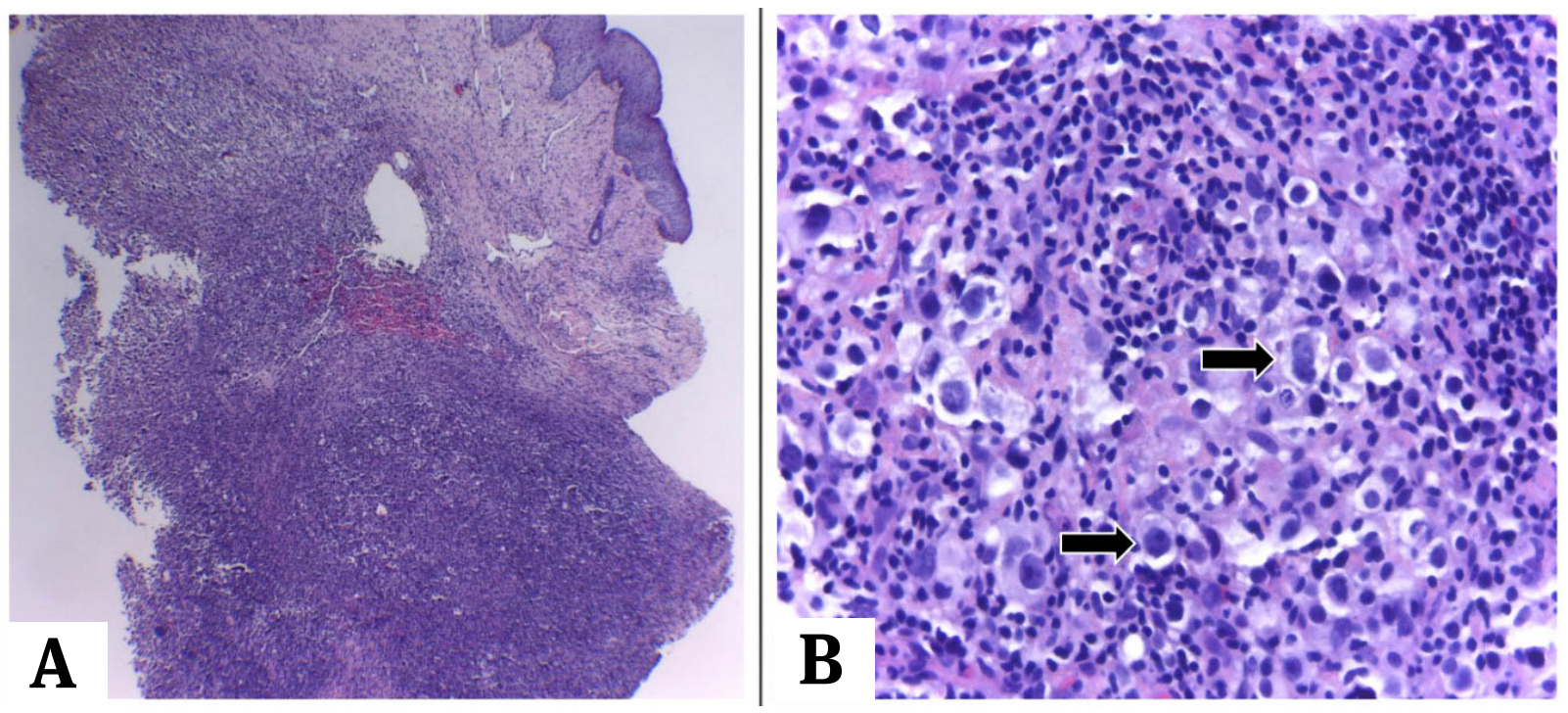

Figura 2. A) Biopsia de piel. A. HE (4X): epidermis sin cambios y en dermis infiltrado difuso de linfocitos pequeños reactivos. B) HE (40X): células grandes atípicas mono y binucleadas compatibles con células de Hodgkin y Reed-Sternberg (flechas). 

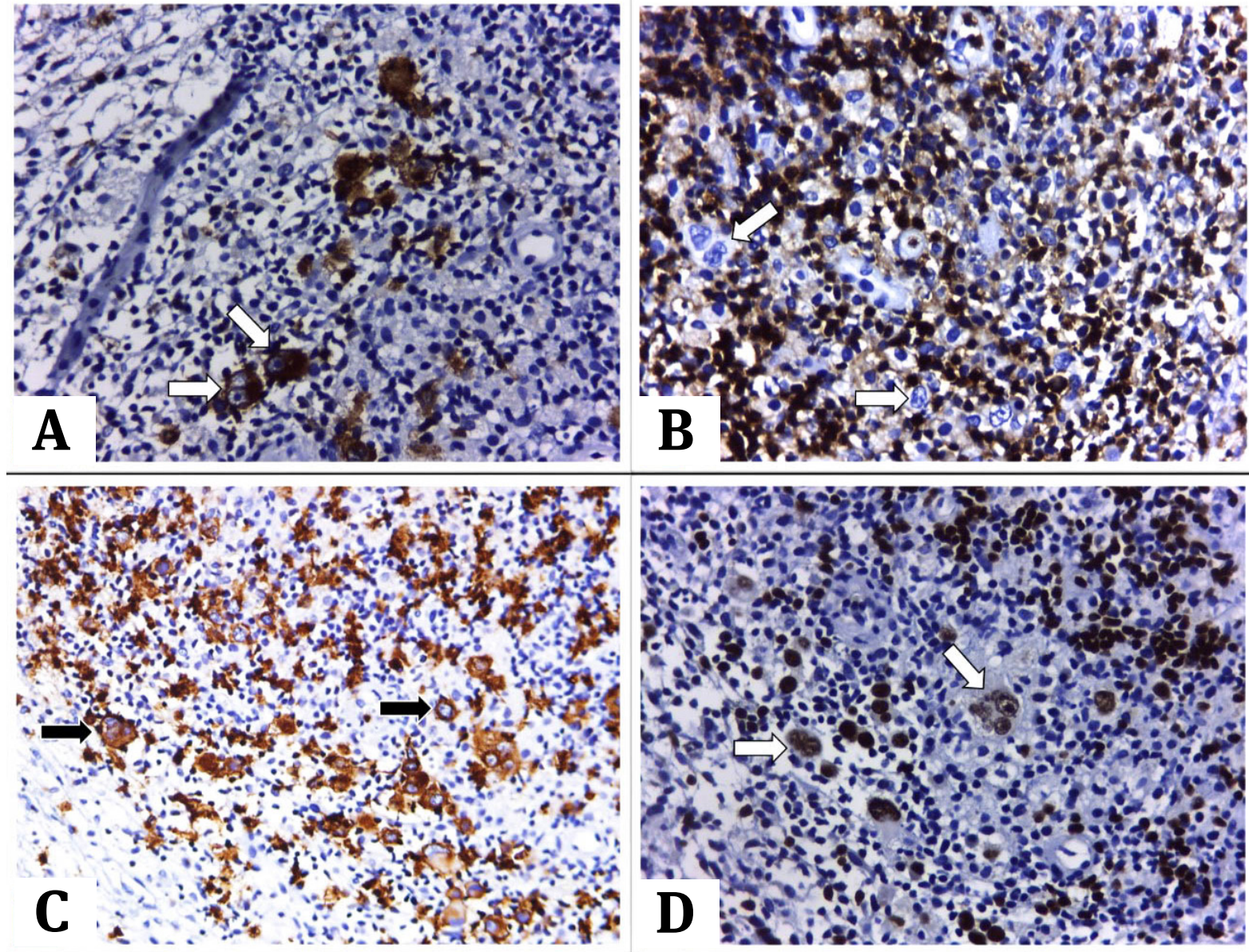

Figura 3. Inmunofenotipo de las células de Hodgkin y Reed-Sternberg (flechas) (40X). A) CD3o positivo. B) CD45 negativo. C) CD15 positivo. D) PAX 15 débil positivo.

morfología y el fenotipo de las células del linfoma y la composición del infiltrado celular: esclerosis nodular (70\%), celularidad mixta (20\%-25\%), rico en linfocitos $(5 \%)$ y con depleción linfoide $(<1 \%)^{(5)}$.

El LH se presenta clínicamente de forma típica como una linfadenopatía indolora, frecuentemente cervical, supraclavicular y/o mediastínica ${ }^{(6)}$. De forma infrecuente puede tener afectación cutánea, descrita por primera vez por el médico alemán Grosz en $1906^{(3)}$.

El compromiso cutáneo tiene dos formas de presentación: la no maligna o paraneoplásica (entre un $17 \%$ y un 53\% de los casos de LH); en esta forma se incluyen diversas manifestaciones inespecíficas como hiperpigmentación, eritrodermia, eccema, prurito, eritema nodoso, eritema multiforme, urticaria e ictiosis; no presenta hallazgos histopatológicos de infiltración de células malignas en la piel ${ }^{(7-9)}$.

La segunda forma cutánea es la maligna o específica, en donde la piel esta histológicamente involucrada, es poco frecuente y se ha reportado entre el o, $5 \%$ y el $7,5 \%$ de los casos de LH. Se manifiesta con lesiones papuloescamosas, nodulares y placas infiltradas pruriginosas en la piel con o sin ulceración ${ }^{(7-9)}$. Puede ocurrir de forma primaria cutánea, en la cual solo hay afectación de la piel y no se evidencia compromiso sistémico de la enfermedad. Es extraordinariamente rara y se ve asociado a un curso indolente; por otro lado, puede manifestarse de forma secundaria a la enfermedad nodal, con aparición simultánea o posterior 
a esta. Ocurre en los estadios avanzados de la enfermedad y es un signo de mal pronóstico ${ }^{(10)}$, como en el caso descrito.

Las lesiones ulcerativas en el LH cutáneo se clasifican en tres tipos:

1. Grosz-Hirschfield: en el cual se desarrolla una ulceración en el nódulo.

2. Coleman-Anderson: donde la ulceración se desarrolla a partir de una infiltración directa en la piel que se extiende desde los ganglios linfáticos subyacentes $\mathrm{u}$ otro tejido.

3. Dossekker-Kren-Saalfeld: que se produce cuando un LH cutáneo primario se ulcera ${ }^{(6)}$.

La afectación específica de la piel por el LH, en su forma secundaria, se puede generar por medio de tres mecanismos: diseminación hematógena, extensión directa desde los ganglios linfáticos o tejidos blandos invadidos previamente por la enfermedad, o diseminación linfática retrógrada desde los ganglios linfáticos proximales; este último mecanismo es el más frecuente y la vía hematógena es la menos frecuente ${ }^{(8,11)}$.

En una serie de 150 pacientes con LH, realizada por Medina y colaboradores, se encontró una diseminación extranodal hacia la piel en 12 de estos pacientes, que presentaron compromiso cutáneo en el territorio del drenaje de los ganglios comprometidos ${ }^{(12)}$.

El diagnóstico del LH cutáneo se realiza por medio de patología e inmunohistoquímica; el LH cutáneo muestra las características del LH nodal con células de Reed-Sternberg, células de Hodgkin y sus variantes, en un fondo de linfocitos, histiocitos, eosinófilos y plasmocitos. Las células de Reed-Sternberg pueden estar ausentes en algunas lesiones cutáneas y también pueden verse en otras condiciones como papulosis linfomatoide o linfoma anaplásico de células grandes ${ }^{(13)}$.

La inmunohistoquímica es determinante en el diagnóstico. Las células neoplásicas de Reed-Stenberg y Hodgkin en el LH clásico son siempre CD30+, CD45-, usualmente CD15+, PAX-5+ débil, en comparación con la población $\mathrm{B}$ reactiva y generalmente negativas para otros marcadores B como CD20 o BOB-1. Los linfocitos acompañantes no tumorales son de linaje B y $\mathrm{T}^{(9,10,13)}$. El VEB puede ser detectado en las células neoplásicas por hibridización in situ (EBER-1) o por inmunohistoquímica para el marcador LMP-1 (proteína latente de membrana) en un grupo de casos; se debe tener presente que esto no es exclusivo de los LH, sino que también puede encontrarse en linfomas no Hodgkin y trastornos linfoproliferativos de células $\mathrm{B}^{(10,11,13)}$. En este caso se realizó inmunohistoquímica para LMP1 en el ganglio linfático, que fue negativa.

Es importante tener en cuenta que la positividad para CD30 no es específica para LH. El CD3o puede expresarse en linfomas cutáneos de células T, como la papulosis linfomatoide y la micosis fungoide, e igualmente en condiciones inflamatorias como reacciones a picaduras de insecto ${ }^{(10,13)}$.

En cuanto al tratamiento, se ha descrito en la literatura que el estándar de oro en LH sin y con compromiso cutáneo es la quimioterapia ABVD (adriamicina, vinblastina, bleomicina y decarbacina) y radioterapia. Este esquema cambia en número de ciclos e intensidad dependiendo del estadio de la enfermedad y de los factores pronósticos del paciente ${ }^{(2,6,7)}$.

Respecto al LH cutáneo primario, la información es limitada debido a los pocos casos reportados; sin embargo, una revisión de 10 casos en la literatura inglesa señala que presenta buena respuesta a tratamientos tales como escisión quirúrgica, terapia con radiación local y/o quimioterapia ABVD ${ }^{(10)}$. Por otro lado, en el LH secundario a enfermedad nodal se emplea quimioterapia ABVD y radioterapia, pero al estar relacionado con estadios avanzados de la enfermedad, tiene mal pronóstico $^{(2,6,7)}$.

\section{CONCLUSIÓN}

Se presenta el caso de un paciente con LH cutáneo secundario a enfermedad nodal, con un estadio avanzado de la enfermedad, que se confirmó por histopatología e inmunopatología. Esta es una variedad poco usual, razón por la cual muchas veces no se sospecha o se diagnostica de forma tardía. Las manifestaciones clínicas no son específicas, por lo que se pueden confundir con otras entidades más frecuentes. Generalmente, las lesiones aparecen cerca de las áreas de la afectación nodal. En nuestro caso, el paciente presentaba lesiones en cara, pero también tenía compromiso de la piel del antebrazo, un sitio distante a los ganglios afectados primariamente. Es importante tener en mente esta afectación cutánea en pacientes con antecedente de LH, para determinar el tratamiento y el pronóstico. 


\section{Puntos clave}

- El LH se presenta clínicamente de forma típica como una linfadenopatía indolora, frecuentemente cervical, supraclavicular y/o mediastínica. De forma infrecuente puede tener afectación cutánea.

- La forma de afectación cutánea específica es inusual, representa entre el o,5\% y el 7,5\% de los casos.

- $\quad$ Ocurre en estadios avanzados de la enfermedad y figura mal pronóstico.

- Se manifiesta con lesiones papuloescamosas, nodulares y placas infiltradas pruriginosas en la piel con o sin ulceración.

- El diagnóstico se confirma por medio de inmunohistoquímica. Las células neoplásicas de Reed-Stenberg y Hodgkin en el LH clásico son siempre CD30+, CD45-, usualmente CD15+, PAX-5+ débil.

\section{REFERENCIAS}

1. Siegel RL, Miller KD, Jemal A. Cancer Statistics, 2017. CA Cancer J Clin. 2017;67(1):7-30. doi: 10.3322/caac. 21442

2. Mugnaini E, Ghosh N. Lymphoma. Prim Care. 2016;43(4):661-75. doi: 10.1016/j.pop.2016.07.012

3. Ambekar AA, Soman CS. Cutaneous Hodgkin's lymphoma. South Asian J Cancer. 2017;6(4):170-5. doi: 10.4103/sajc.sajc_195_17

4. Murray PG, Young LS. An etiological role for the Epstein-Barr virus in the pathogenesis of classical Hodgkin lymphoma. Blood. 2019;134(7):591-6. doi: 10.1182/blood.2019000568

5. Jianga M, Bennanib N, Feldmana A. Lymphoma classification update: T-cell lymphomas, Hodgkin lymphomas, and histiocytic/dendritic cell neoplasms. Expert Rev Hematol. 2017;10(3):239-49. doi: 10.1080/17474086.2017.1281122

6. Khalifeh I, Hughey LC, Huang CC, Reddy VVB, Sellheyer K. Solitary plaque on the scalp as a primary manifestation of Hodgkin lymphoma: a case report and review of the literature. J Cutan Pathol. 2009;36(Suppl. 1):80-5. doi: 10.1111/j.1600-0560.2009.01227.x

7. Oluwolea O, Zic J, Douds JA, Thompson MA, Greer JP. Cutaneous manifestations and management of hematologic neo- plasms. Semin Oncol. 2016;43(3):370-83. doi: 10.1053/j.seminoncol.2016.02.021

8. Serra-Guillén C, Herrero J, Requena C, Alfaro A, Sanmartín O, Nagore E, Botella R, Guillén C. Afectación cutánea específica por enfermedad de Hodgkin. Actas Dermosifiliogr. 2004;95(9):571-3.

9. Pranteda G, Osti MF, Cox MC, Cacchi C, Carlesimo M, Grimaldi M, Fidanza L, Bartolazzi A. Primary cutaneous Hodgkin lymphoma. J Am Acad Dermatol. 2010;63(2):52-3. doi: 10.1016/j.jaad.2009.06.076

10. Huong G, Olson L, Rippis G, Magro C. An Index Case of Cutaneous Hodgkin Lymphoma and Review of the Literature. Am J Dermatopathol. 2016;38(10):739-43. doi: 10.1097/DAD.0000000000000543

11. Mukesh M, Shuttleworth D, Murray P. Primary cutaneous Hodgkin's lymphoma. Clin Exp Dermatol. 2009;34(8):e673-5. doi: 10.1111/j.1365-2230.2009.03402.x

12. Medina A, Benninghoff DL, Camiel MR. Extra nodal spread of Hodgkin's disease. Am J Roentgenol Radium Ther Nucl Med. 1971;111(2):368-75. doi: 10.2214/ajr.111.2.368

13. Cerroni L. Cutaneous manifestations of Hodgkin lymphoma. Skin lymphoma. 4.a edición. Austria: Wiley-Blackwell; 2014. p. 331-40. 